\title{
Kaluza-Klein black lens in five dimensions
}

\author{
Shinya Tomizawa* \\ Department of Liberal Arts, Tokyo University of Technology, \\ 5-23-22, Nishikamata, Otaku, Tokyo 144-8535, Japan
}

(Received 31 March 2018; published 5 July 2018)

\begin{abstract}
We obtain a supersymmetric Kaluza-Klein black lens solution in Taub-NUT space in the fivedimensional minimal ungauged supergravity. It is shown that the spacetime has a degenerate horizon with the spatial cross section of the lens space topology $L(n, 1)=S^{3} / \mathbb{Z}_{n}$ and looks like the four-dimensional Minkowski spacetime in the neighborhood of spatial infinity. In contrast to the horizon topology, from a five-dimensional point of view, the spatial infinity has the topology of $S^{3}$ rather than the lens space, for which this solution has an asymptotically flat limit. We discuss several properties of such a black lens, in particular, the effect by the compactification of an extra dimension and some physical differences from the asymptotically flat supersymmetric black lens, which has recently been found.
\end{abstract}

DOI: 10.1103/PhysRevD.98.024012

\section{INTRODUCTION}

Higher-dimensional black holes/rings and other extended black objects have been considered to play essential roles in the various contexts of the statistical counting of black-hole entropy, the AdS/CFT correspondence, and the black-hole production at an accelerator. In particular, physics of black holes in five-dimensional Einstein-Maxwell-Chern-Simons (EMCS) theory has recently been the subject of increased attention, since the five-dimensional EMCS theory describes the bosonic sector of five-dimensional minimal supergravity as a low-energy limit of string theory as well as one of the simplest theories of supersymmetry. So far, several types of black-hole solutions in this theory have been found by using the recent development of solution-generating techniques [1-9], and they have been classified by its uniqueness theorems [10-12]. However, it is evident that the construction of all black-hole solutions has not been achieved yet.

It is now evident that, even in vacuum Einstein theory, there is a much richer variety of black-hole solutions in higher dimensions. For instance, the topology theorem [13-16] for stationary black holes, which is generalized to five dimensions, allows the topology of the spatial cross section of the event horizon to be either a sphere $S^{3}$, a ring $S^{1} \times S^{2}$, or lens spaces $L(p, q)$ under the assumptions of asymptotic flatness and biaxisymmetry. Moreover, as for supersymmetric solutions in five-dimensional minimal

\footnotetext{
tomizawasny@stf.teu.ac.jp
}

Published by the American Physical Society under the terms of the Creative Commons Attribution 4.0 International license. Further distribution of this work must maintain attribution to the author(s) and the published article's title, journal citation, and DOI. Funded by SCOAP ${ }^{3}$. supergravity, it has recently been shown that under the same symmetry assumptions, the horizon topology must be one of $S^{3}, S^{1} \times S^{2}$ or lens spaces $L(p, 1)$, which rules out $L(p, q)(q \neq 1)$ [17]. As for both of the sphere $S^{3}$ and the ring topology $S^{1} \times S^{2}$, the corresponding exact solutions have been found as stationary solutions to the five-dimensional vacuum Einstein equations [18-21]. However, for the lens space topologies $L(p, q)$, they have not yet been found, in spite of the efforts of some researchers to construct them as a regular vacuum solution $[22,23]$.

Recently, however, within the class of the supersymmetric solutions in the bosonic sector of the fivedimensional minimal ungauged supergravity, the first regular black lens solution with asymptotic flatness has been constructed by Kunduri and Lucietti [24] for the horizon topology of $L(2,1)=S^{3} / \mathbb{Z}_{2}$. Thereafter, this was generalized to the supersymmetric solution with the horizon of the more general lens space topologies $L(p, 1)=$ $S^{3} / \mathbb{Z}_{p}(p \geq 3)$ by the author of this paper and Nozawa [25]. How to construct these solutions is based on the wellknown work of the classification of supersymmetric solutions by Gauntlett et al. [1]. Moreover, this has been immediately extended to a multi-black lens solution [26] and a cosmological black lens solution [27].

The assumption of asymptotic flatness is mainly related to the context of a braneworld model, in which the size of higher-dimensional black holes can become much smaller than the size of extra dimensions. However, since our visible world is thought to be macroscopically four dimensional, extra dimensions must be compactified in some sense. In this direction, it has been considered to be of great interest to consider higher-dimensional Kaluza-Klein black holes since they look like four-dimensional ones, at least, 
at infinity, although they appear higher dimensional near the horizon [28-36]. Such Kaluza-Klein solutions can be expected to help us to get some insights into the major open problem about how to compactify and stabilize extra dimensions in string theory. The main purpose of this paper is to construct a certain type of supersymmetric KaluzaKlein black lens solutions in five-dimensional minimal supergravity, understanding the novel effect by the compactification of an extra dimension and making it clear what is essentially different from the asymptotic flat black lens solutions.

It is mathematically well known that the lens spaces $L(p, q)$ (where $p$ and $q$ are coprime integers) are quotients of $S^{3}$ by $\mathbb{Z} / p$ action, which can be regarded as an $S^{1}$ fiber bundle over an $S^{2}$. In particular, the regular metric on the lens space $L(p, 1)=S^{3} / \mathbb{Z}_{p}$ is simply written as

$d s^{2}=\frac{1}{4}\left[R_{1}^{2}\left(\frac{d \psi}{p}+\cos \theta d \phi\right)^{2}+R_{2}^{2}\left(d \theta^{2}+\sin ^{2} \theta d \phi^{2}\right)\right]$,

where $0 \leq \psi<4 \pi, 0 \leq \phi<2 \pi, 0 \leq \theta \leq \pi$, and $R_{1} / 2$, $R_{2} / 2$ are the radii of the $S^{1}$ and $S^{2}$, respectively. When $p=1$, this reduces to the metric on an $S^{3}$ written in terms of the Euler angles, which is often referred to as round $S^{3}$ for $R_{1}=R_{2}$ and squashed $S^{3}$ for $R_{1} \neq R_{2}$. An asymptotically flat black lens spacetime [24,25] has the spatial infinity of a round $S^{3}$, where the ratio $R_{2} / R_{1}$ is 1 . In this paper, we would like to consider an asymptotically KaluzaKlein black lens spacetime with the spatial infinity of a squashed $S^{3}[p=1$ in Eq. (1)] and a horizon of the lens space $L(n, 1)$ [ $p=n$ in Eq. (1)], where the size of an $S^{1}$ is much smaller than that of an $S^{2}$ (i.e., $R_{1} / R_{2} \rightarrow 0$ ) at infinity and $R_{1}$ and $R_{2}$ are finite on the horizon. Therefore, we will impose the appropriate boundary conditions on the parameters included in the supersymmetric solutions on the Taub-NUT space.

This paper is organized as follows. In Sec. II, we give the supersymmetric black lens solutions with bubbles in TaubNUT space in the five-dimensional minimal ungauged supergravity. In Sec. III, we impose the boundary conditions so that the spacetime is asymptotically KaluzaKlein spacetime and has no closed timelike curves (CTCs) appear around the horizon, no conical nor curvature singularities in the domain of outer communications, and no orbifold singularities nor Dirac-Misner strings on the axis. In Sec. IV, we discuss some physical properties of such a black lens. In Sec. V, we devote ourselves to the summary and discussion on our results.

\section{SOLUTION}

We consider supersymmetric solutions in the fivedimensional minimal ungauged supergravity, the bosonic Lagrangian of which consists of the Einstein-Maxwell theory with a Chern-Simons term and takes the form [1]

$$
\mathcal{L}=R \star 1-2 F \wedge \star F-\frac{8}{3 \sqrt{3}} A \wedge F \wedge F,
$$

where $F=d A$ is the Maxwell field. The local metric and the gauge potential 1-form of a supersymmetric KaluzuaKlein black lens solution in this theory have the form

$$
\begin{gathered}
d s^{2}=-f^{2}(d t+\omega)^{2}+f^{-1} d s_{M}^{2}, \\
A=\frac{\sqrt{3}}{2}\left[f(d t+\omega)-\frac{K}{H}(d \psi+\chi)-\xi\right],
\end{gathered}
$$

where $d s_{M}^{2}$ is the metric on the Gibbons-Hawking space [37],

$d s_{M}^{2}=H^{-1}(d \psi+\chi)^{2}+H d x^{i} d x^{i}, \quad d \chi=* d H$,

where $\left\{x^{i}\right\}=(x, y, z)(i=1,2,3)$ are Cartesian coordinates on $\mathbb{E}^{3}$. The triholomorphic Killing vector $\partial / \partial \psi$ continues a symmetry generator for the five-dimensional metric $g_{\mu \nu}$ and the gauge field $A_{\mu}$, Furthermore, every bosonic element can be obtained as [1]

$$
\begin{gathered}
f^{-1}=H^{-1} K^{2}+L, \\
\omega=\omega_{\psi}(d \psi+\chi)+\hat{\omega}, \\
\omega_{\psi}=H^{-2} K^{3}+\frac{3}{2} H^{-1} K L+M, \\
* d \hat{\omega}=H d M-M d H+\frac{3}{2}(K d L-L d K), \\
d \xi=-* d K .
\end{gathered}
$$

Following Refs. [24,25], we consider the following class of harmonic functions:

$$
\begin{gathered}
H=h_{0}+\sum_{i=1}^{n} \frac{h_{i}}{r_{i}}:=h_{0}+\frac{n}{r_{1}}-\sum_{i=2}^{n} \frac{1}{r_{i}}, \\
M=m_{0}+\sum_{i=1}^{n} \frac{m_{i}}{r_{i}}, \\
K=k_{0}+\sum_{i=1}^{n} \frac{k_{i}}{r_{i}}, \\
L=l_{0}+\sum_{i=1}^{n} \frac{l_{i}}{r_{i}} .
\end{gathered}
$$

Here, $\quad r_{i}:=\left|\boldsymbol{r}-\boldsymbol{r}_{i}\right|=\sqrt{\left(x-x_{i}\right)^{2}+\left(y-y_{i}\right)^{2}+\left(z-z_{i}\right)^{2}}$, where $\left(x_{i}, y_{i}, z_{i}\right)$ are constants. 
The 1 -forms $(\chi, \xi, \hat{\omega})$ are obtained as

$$
\begin{gathered}
\chi=\sum_{i=1}^{n} h_{i} \tilde{\omega}_{i}, \\
\xi=-\sum_{i=1}^{n} k_{i} \tilde{\omega}_{i}, \\
\hat{\omega}=\sum_{i, j=1(i \neq j)}^{n}\left(h_{i} m_{j}+\frac{3}{2} k_{i} l_{j}\right) \hat{\omega}_{i j} \\
-\sum_{i=1}^{n}\left(m_{0} h_{i}+\frac{3}{2} l_{0} k_{i}-h_{0} m_{i}\right) \tilde{\omega}_{i},
\end{gathered}
$$

where 1 -forms $\tilde{\omega}_{i}$ and $\hat{\omega}_{i j}(i \neq j)$ on $\mathbb{E}^{3}$ are defined by (cf. Ref. [38]),

$$
\begin{aligned}
\hat{\omega}_{i j}= & -\left(\frac{\left(\boldsymbol{r}-\boldsymbol{r}_{i}\right) \cdot\left(\boldsymbol{r}-\boldsymbol{r}_{j}\right)}{r_{i} r_{j}}+c_{i j}\right) \\
& \times \frac{\left[\left(\boldsymbol{r}_{i}-\boldsymbol{r}_{j}\right) \times\left(\boldsymbol{r}-\frac{\boldsymbol{r}_{i}+\boldsymbol{r}_{j}}{2}\right)\right]_{k} d x^{k}}{\left|\left(\boldsymbol{r}_{i}-\boldsymbol{r}_{j}\right) \times\left(\boldsymbol{r}-\frac{\boldsymbol{r}_{i}+\boldsymbol{r}_{j}}{2}\right)\right|^{2}}, \\
\tilde{\omega}_{i}= & \frac{z-z_{i}}{r_{i}} \frac{\left(x-x_{i}\right) d y-\left(y-y_{i}\right) d x}{\left(x-x_{i}\right)^{2}+\left(y-y_{i}\right)^{2}},
\end{aligned}
$$

where $c_{i j}=-c_{j i}$ are constants and $z_{j i}:=z_{j}-z_{i}$. Throughout this paper, we set $x_{i}=y_{i}=0$ for all $i$ (in this case, $x \partial / \partial y-y \partial / \partial x$ is another Killing field) and assume $z_{i}<z_{j}$ for $i<j$. In this case, $\hat{\omega}$ and $\tilde{\omega}_{i}$ can be written in spherical coordinates by $x=r \sin \theta \cos \phi$, $y=\sin \theta \sin \phi, z=r \cos \theta$ as

$$
\begin{aligned}
\hat{\omega}= & {\left[\sum_{i, j=1(i \neq j)}^{n}\left(h_{i} m_{j}+\frac{3}{2} k_{i} l_{j}\right) \frac{r^{2}-\left(z_{i}+z_{j}\right) r \cos \theta+z_{i} z_{j}}{z_{j i} r_{i} r_{j}}\right.} \\
& \left.-\sum_{i=1}^{n}\left(m_{0} h_{i}+\frac{3}{2} l_{0} k_{i}-h_{0} m_{i}\right) \frac{r \cos \theta-z_{i}}{r_{i}}+c\right] d \phi,
\end{aligned}
$$

with the constant $c^{1}$ and $z_{j i}:=z_{j}-z_{i}$.

It should be noted that there exists a gauge freedom of redefining harmonic functions [39]

$$
\begin{aligned}
& K \rightarrow K+a H, \quad L \rightarrow L-2 a K-a^{2} H, \\
& M \rightarrow M-\frac{3}{2} a L+\frac{3}{2} a^{2} K+\frac{1}{2} a^{3} H
\end{aligned}
$$

\footnotetext{
${ }^{1}$ We have put $c:=\sum_{i, j=1(i \neq j)}^{n}\left(h_{i} m_{j}+\frac{3}{2} k_{i} l_{j}\right) \frac{c_{i j}}{z_{j i}}$.
}

where $a$ is a constant. Under the transformation (21), the constant term $k_{0}$ in $K$ changes as $k_{0} \rightarrow k_{0}+a h_{0}$. In an appropriate choice of $a$, one can put

$$
k_{0}=0 \text {. }
$$

In the limit of $h_{0} \rightarrow 0$, this solution reduces to the asymptotically flat black lenses [25] (in the appropriate choice of the parameters), the Breckenridge-Myers-PeetVafa (BMPV) black hole [40] with the horizon of spherical topology for $n=1$, and the supersymmetric black lens with the horizon of the lens space topology $L(2,1)=S^{3} / \mathbb{Z}_{2}$ of Kunduri and Lucietti [24] for $n=2$.

\section{BOUNDARY CONDITIONS}

To obtain a supersymmetric Kaluza-Klein black lens solution of physical interest, we impose boundary conditions at spacetime boundaries, at infinity $r \rightarrow \infty$, on the horizon $\boldsymbol{r}=\boldsymbol{r}_{1}$, at the points $\boldsymbol{r}=\boldsymbol{r}_{i}(i=2, \ldots, n)$, and on the $z$ axis $x=y=0$ in $\mathbb{E}^{3}$ of the Gibbons-Hawking base space:

(i) (i) At infinity $r \rightarrow \infty$, the extra dimension of the spacetime is compactified so that the size of the fifth dimension is much smaller than that of the other spatial dimensions. Hence, the spacetime can be asymptotically approximated as an $S^{1}$ fiber bundle over four-dimensional Minkowski spacetime. Moreover, we make an additional assumption that spatial infinity is topologically $S^{3}$ so that the obtained solution can have a limit at an asymptotically flat solution.

(ii) (ii) At the horizon $\boldsymbol{r}=\boldsymbol{r}_{1}$, the surface should be a smooth degenerate null surface of which the spatial cross section has a topology of the lens space $L(n, 1)=S^{3} / \mathbb{Z}_{n}$.

(iii) (iii) At the $(n-1)$ points $\boldsymbol{r}=\boldsymbol{r}_{i}(i=2, \ldots, n)$, where each harmonic function diverges, the metric behaves like the origin of the Minkowski spacetime.

(iv) (iv) On the $z$ axis in $\mathbb{E}^{3}$ of the Gibbons-Hawking base space, there exist no Dirac-Misner strings nor orbifold singularities.

Furthermore, under these boundary conditions, the spacetime is required to allow neither CTCs nor (conical and curvature) singularities.

\section{A. Infinity}

For $r \rightarrow \infty$, the metric functions $f$ and $\omega_{\psi}$ behave, respectively, as

$$
f^{-1} \simeq l_{0}+\frac{\sum_{i} l_{i}}{r}, \quad \omega_{\psi} \simeq m_{0}+\frac{\frac{3}{2} l_{0} \sum_{i} k_{i}+\sum_{i} m_{i}}{r} .
$$

The 1 -forms $\omega$ and $\chi$ behave, respectively, as

$$
\chi \simeq \cos \theta d \phi,
$$




$$
\omega \simeq m_{0}\left(d \psi+\sum_{i} h_{i} \cos \theta d \phi\right)-\sum_{i}\left(m_{0} h_{i}+\frac{3}{2} l_{0} k_{i}-h_{0} m_{i}\right) \cos \theta d \phi+\left(\sum_{i, j(i \neq j)} \frac{h_{i} m_{j}+\frac{3}{2} k_{i} l_{j}}{z_{j i}}+c\right) d \phi
$$

Therefore, the metric can be approximated as

$$
\begin{aligned}
d s^{2} \simeq & -l_{0}^{-2}\left[d t+m_{0}(d \psi+\cos \theta d \phi)-\sum_{i}\left(m_{0} h_{i}+\frac{3}{2} l_{0} k_{i}-h_{0} m_{i}\right) \cos \theta d \phi+\left(\sum_{i, j(i \neq j)} \frac{h_{i} m_{j}+\frac{3}{2} k_{i} l_{j}}{z_{j i}}+c\right) d \phi\right]^{2} \\
& +l_{0}\left[h_{0}^{-1}\left(d \psi+\sum_{i} h_{i} \cos \theta d \phi\right)^{2}+h_{0}\left\{d r^{2}+r^{2}\left(d \theta^{2}+\sin ^{2} \theta d \phi^{2}\right)\right\}\right] .
\end{aligned}
$$

The boundary condition (i) at infinity demands the parameters satisfy

$$
\begin{gathered}
l_{0}=1, \\
\sum_{i}\left(m_{0} h_{i}+\frac{3}{2} l_{0} k_{i}-h_{0} m_{i}\right)=0, \\
c=-\sum_{i, j(i \neq j)} \frac{h_{i} m_{j}+\frac{3}{2} k_{i} l_{j}}{z_{j i}} .
\end{gathered}
$$

In the choice of these parameters, for $r \rightarrow \infty$, the metric asymptotically becomes

$$
\begin{aligned}
d s^{2} \simeq & -\left[d t+m_{0}(d \psi+\cos \theta d \phi)\right]^{2} \\
& +h_{0}\left[d r^{2}+r^{2}\left(d \theta^{2}+\sin ^{2} \theta d \phi^{2}\right)\right] \\
& +h_{0}^{-\frac{1}{2}}(\psi+\cos \theta d \phi)^{2} \\
= & -\left[d t+m_{0}(d \psi+\cos \theta d \phi)\right]^{2}+d \bar{r}^{2} \\
+ & \bar{r}^{2}\left(d \theta^{2}+\sin ^{2} \theta d \phi^{2}\right)+\left(d w+h_{0}^{-\frac{1}{2}} \cos \theta d \phi\right)^{2},
\end{aligned}
$$

where we have defined $\bar{r}=h_{0}^{\frac{1}{2}} r$ and $w=h_{0}^{-\frac{1}{2}} \psi$. Because of the presence of the cross term $-m_{0}(d \psi+\cos \theta d \phi) d t$, this does not seem to satisfy boundary condition (i), but this is because the asymptotic form is not at the rest frame. Therefore, to move to the rest frame, let us define the coordinates $(\bar{t}, \bar{w})$ by

$$
t=\sqrt{1-h_{0} m_{0}^{2}} \bar{t}, \quad w=\frac{\bar{w}+h_{0}^{-\frac{1}{2}} m_{0} \bar{t}}{\sqrt{1-h_{0} m_{0}^{2}}}
$$

In terms of these new coordinates, the asymptotic metric can be rewritten as

$$
\begin{aligned}
d s^{2} \simeq & -d t^{2}+d \bar{r}^{2}+\bar{r}^{2}\left(d \theta^{2}+\sin ^{2} \theta d \phi\right)^{2} \\
& +\left(d \bar{w}+h_{0}^{-\frac{1}{2}} \cos \theta d \phi\right)^{2} .
\end{aligned}
$$

This is the metric of an $S^{1}$ fiber bundle over Minkowski spacetime, where the radius of $S^{2}$ becomes infinite but that of $S^{1}$ remains finite. The avoidance of conical singularities requires the range of angles to be $0 \leq \theta \leq \pi, 0 \leq \phi<2 \pi$, and $0 \leq \psi<4 \pi$ with the identification $\phi \sim \phi+2 \pi$ and $\psi \sim \psi+4 \pi$, under which assumptions the spatial infinity is topologically an $S^{3}$ rather than a lens space. From $w \sim w+2 \pi R_{k}, \bar{w} \sim \bar{w}+2 \pi R_{5}$, we can observe that the radius of the Kaluza-Klein circle $R_{5}$ at infinity is

$$
R_{5}=\sqrt{1-h_{0} m_{0}^{2}} R_{k}=\frac{2 \sqrt{1-h_{0} m_{0}^{2}}}{\sqrt{h_{0}}}
$$

In the limit $h_{0} \rightarrow 0$, the Kaluza-Klein circle $R_{5}$, namely, the size of an extra dimension becomes infinite, which corresponds to the asymptotically flat black lens $[24,25]$.

\section{B. Horizon $r=r_{1}$}

Now, we see that the point source $\boldsymbol{r}=\boldsymbol{r}_{1}$ corresponds to a degenerate Killing horizon of which the topology of the spatial cross section is the lens space of $L(n, 1)=S^{3} / \mathbb{Z}_{n}$. Since, without loss of generality, we can choose the point source $\boldsymbol{r}_{1}$ as the origin of $\mathbb{E}^{3}$ in the Gibbons-Hawking base space, we consider here only the geometry near the origin $r=0$. Around this point, the functions $f^{-1}$ and $\omega_{\psi}$ are expanded as

$$
\begin{aligned}
f^{-1} & \simeq \frac{k_{1}^{2} / h_{1}+l_{1}}{r}+c_{1}^{\prime}, \\
\omega_{\psi} & \simeq \frac{k_{1}^{3} / h_{1}^{2}+3 k_{1} l_{1} / 2 n+m_{1}}{r}+c_{2}^{\prime},
\end{aligned}
$$

where we have defined the constants $c_{1}^{\prime}$ and $c_{2}^{\prime}$ by

$$
c_{1}^{\prime}:=l_{0}-\frac{h_{0}}{h_{1}^{2}} k_{1}^{2}+\sum_{i \neq 1} \frac{1}{h_{1}^{2}\left|z_{i 1}\right|}\left[2 h_{1} k_{1} k_{i}-k_{1}^{2} h_{i}+h_{1}^{2} l_{i}\right]
$$




$$
c_{2}^{\prime}:=m_{0}+\frac{3}{2 h_{1}^{2}}\left(h_{1} k_{1} l_{0}-h_{0} k_{1} l_{1}\right)-\frac{2 h_{0} k_{1}^{3}}{h_{1}^{3}}+\sum_{i \neq 1} \frac{1}{2 h_{1}^{3}\left|z_{i 1}\right|}\left[-\left(4 k_{1}^{3}+3 h_{1} k_{1} l_{1}\right) h_{i}+3 h_{1}\left(2 k_{1}^{2}+h_{1} l_{1}\right) k_{i}+3 h_{1}^{2} k_{1} l_{i}+2 h_{1}^{3} m_{i}\right]
$$

The 1 -forms $\omega$ and $\chi$ behave, respectively, as

$$
\begin{aligned}
\hat{\omega}= & {\left[\sum_{j \neq 1}\left(n m_{j}+\frac{3}{2} k_{1} l_{j}-\frac{3}{2} k_{j} l_{1}\right) \frac{-\cos \theta}{\left|z_{j 1}\right|}+\sum_{i, j \neq 1(i \neq j)}\left(-m_{j}+\frac{3}{2} k_{i} l_{j}\right) \frac{z_{i 1} z_{j 1}}{\left|z_{i 1} z_{j 1}\right| z_{j i}}\right.} \\
& \left.-\left(m_{0} n+\frac{3}{2} l_{0} k_{1}-h_{0} m_{1}\right) \cos \theta-\sum_{i \neq 1}\left(-m_{0}+\frac{3}{2} l_{0} k_{i}-h_{0} m_{i}\right) \frac{-z_{i 1}}{\left|z_{i 1}\right|}+c\right] d \phi
\end{aligned}
$$

and

$$
\chi=h_{1} \hat{\omega}_{1}+\sum_{i \neq 1} h_{i} \hat{\omega}_{i} \simeq\left(n \cos \theta+\sum_{i \neq 1} \frac{z_{i 1}}{\left|z_{i 1}\right|}\right) d \phi .
$$

In terms of new coordinates $\left(v, \psi^{\prime}\right)$ defined by

$$
\begin{aligned}
d v & =d t-\left(\frac{A_{0}}{r^{2}}+\frac{A_{1}}{r}\right) d r \\
d \psi^{\prime} & =d \psi+\sum_{i \neq 1} \frac{z_{i 1}}{\left|z_{i 1}\right|} d \phi-\frac{B_{0}}{r} d r
\end{aligned}
$$

we can confirm that the divergences of $g_{r r}$ and $g_{r \psi^{\prime}}$ can be eliminated and the metric is analytic at $\boldsymbol{r}=0$, where the constants $A_{0}, A_{1}$, and $B_{0}$ are defined by

$$
A_{0}=\frac{1}{2} \sqrt{3 k_{1}^{2} l_{1}^{2}+4 h_{1} l_{1}^{3}-4 m_{1}\left(2 k_{1}^{3}+3 h_{1} k_{1} l_{1}+h_{1}^{2} m_{1}\right)},
$$

$$
A_{0} B_{0}=\frac{2 k_{1}^{3}+3 h_{1} k_{1} l_{1}+2 h_{1}^{2} m_{1}}{2}
$$

Hence, it turns out that the point $\boldsymbol{r}=\boldsymbol{r}_{1}$ corresponds to the Killing horizon for the supersymmetric Killing field $V=\partial / \partial v$. Moreover, after putting $(v, r) \rightarrow(v / \epsilon, \epsilon r)$, taking the limit of $\epsilon \rightarrow 0$, we can obtain the near-horizon geometry

$$
\begin{aligned}
d s_{\mathrm{NH}}^{2}= & \frac{R_{2}^{2}}{4}\left[d \psi^{\prime}+n \cos \theta d \phi\right. \\
& \left.-\frac{2 k_{1}\left(2 k_{1}^{2}+3 n l_{1}\right)+4 n^{2} m_{1}}{R_{1}^{4} R_{2}^{2}} r d v\right]^{2} \\
& +R_{1}^{2}\left(d \theta^{2}+\sin ^{2} \theta d \phi^{2}\right) \\
& -\frac{4 r^{2}}{R_{1}^{2} R_{2}^{2}} d v^{2}-\frac{4}{R_{2}} d v d r
\end{aligned}
$$

where we have defined

$$
R_{1}^{2}:=k_{1}^{2}+n l_{1}
$$

$R_{2}^{2}:=\frac{l_{1}^{2}\left(3 k_{1}^{2}+4 n l_{1}\right)-4 m_{1}\left(2 k_{1}^{3}+3 n k_{1} l_{1}+n^{2} m_{1}\right)}{R_{1}^{4}}$

This metric is locally isometric to the near-horizon geometry of the BMPV black hole [41]. To eliminate CTCs around the horizon, we must require

$$
R_{1}^{2}>0
$$

and

$$
R_{2}^{2}>0
$$

The spatial cross section of the event horizon can be extracted by $v=$ const and $r=0$ in (44), 
$d s_{\mathrm{H}}^{2}=\frac{R_{2}^{2} n^{2}}{4}\left(\frac{d \psi^{\prime}}{n}+\cos \theta d \phi\right)^{2}+R_{1}^{2}\left(d \theta^{2}+\sin ^{2} \theta d \phi^{2}\right)$,

which is the squashed metric of the lens space $S^{3} / \mathbb{Z}_{n}$.

\section{Points $r=r_{i}(n=2, \ldots, n)$}

The metric of the Gibbons-Hawking space has apparent divergences at the points $\boldsymbol{r}=\boldsymbol{r}_{i}(n=2, \ldots, n)$, but it can be shown that they correspond to coordinate singularities under the appropriate parameter setting. We impose the boundary conditions so that each point $\boldsymbol{r}=\boldsymbol{r}_{i}(n=2, \ldots, n)$ behaves like the smooth origin of Minkowski spacetime. Let us choose the coordinates $\boldsymbol{r}=(x, y, z)$ on $\mathbb{E}^{3}$ in the Gibbons-Hawking space so that the $i$ th point $\boldsymbol{r}_{i}(i \neq 1)$ becomes the origin of $\mathbb{E}^{3}$ (i.e., $\boldsymbol{r} \rightarrow \boldsymbol{r}-\boldsymbol{r}_{i}$ ), near which point, the functions $f^{-1}$ and $\omega_{\psi}$ behave, respectively, as

$$
f^{-1} \simeq \frac{l_{i}+\frac{k_{i}^{2}}{h_{i}}}{r}+c_{1}, \quad \omega_{\psi} \simeq \frac{\frac{k_{i}^{3}}{h_{i}^{2}}+\frac{3}{2 h_{i}} k_{i} l_{i}+m_{i}}{r}+c_{2},
$$

where the constants $c_{1}$ and $c_{2}$ are defined by

$$
\begin{aligned}
c_{1}:= & l_{0}+\frac{-h_{0} k_{i}^{2}}{h_{i}^{2}}+\sum_{j(\neq i)} \frac{1}{h_{i}^{2}\left|z_{j i}\right|}\left(-k_{i}^{2} h_{j}+2 h_{i} k_{i} k_{j}+h_{i}^{2} l_{j}\right), \\
c_{2}:= & m_{0}+\frac{1}{2 h_{i}^{3}}\left(-4 h_{0} k_{i}^{3}+3 h_{i}^{2} k_{i} l_{0}-3 h_{0} h_{i} k_{i} l_{i}\right) \\
& +\sum_{j(\neq i)} \frac{1}{2 h_{i}^{3}\left|z_{j i}\right|}\left[-\left(4 k_{i}^{3}+3 h_{i} k_{i} l_{i}\right) h_{j}\right. \\
& \left.+3 h_{i}\left(2 k_{i}^{2}+h_{i} l_{i}\right) k_{j}+3 h_{i}^{2} k_{i} l_{j}+2 h_{i}^{3} m_{j}\right] .
\end{aligned}
$$

The 1 -forms $\chi$ and $\hat{\omega}$ are approximated as

$$
\chi \simeq\left(h_{i} \cos \theta+\chi_{(0)}\right) d \phi, \quad \hat{\omega} \simeq\left(\hat{\omega}_{(1)} \cos \theta+\hat{\omega}_{(0)}\right) d \phi,
$$

where

$$
\chi_{(0)}:=-\sum_{j(\neq i)} \frac{h_{j} z_{j i}}{\left|z_{j i}\right|},
$$

$$
\begin{aligned}
\hat{\omega}_{(0)}:= & \sum_{k, j \neq i, k \neq j)}\left(h_{k} m_{j}+\frac{3}{2} k_{k} l_{j}\right) \frac{z_{j i} z_{k i}}{\left|z_{j i} z_{k i}\right| z_{j k}} \\
& +\sum_{j(\neq i)}\left(m_{0} h_{j}+\frac{3}{2} k_{j} l_{0}-h_{0} m_{j}\right) \frac{z_{j i}}{\left|z_{j i}\right|}+c,
\end{aligned}
$$

$$
\begin{aligned}
\hat{\omega}_{(1)}:= & -\sum_{j(\neq i)}\left(h_{i} m_{j}-h_{j} m_{i}+\frac{3}{2}\left(k_{i} l_{j}-k_{j} l_{i}\right)\right) \frac{1}{\left|z_{j i}\right|} \\
& -\left(m_{0} h_{i}+\frac{3}{2} k_{i} l_{0}-h_{0} m_{i}\right) .
\end{aligned}
$$

Therefore, the asymptotic behavior of the metric around this point can be written as

$$
\begin{aligned}
d s^{2} \simeq & -\left(\frac{k_{i}^{2} / h_{i}+l_{i}}{r}+c_{1}\right)^{-2}\left[d t+\left(\frac{k_{i}^{3} / h_{i}^{2}+\frac{3}{2} k_{i} l / h_{i}+m_{i} / h_{i}^{2}}{r}+c_{2}\right)\left\{d \psi+\left(h_{i} \cos \theta+\chi_{(0)}\right) d \phi\right\}+\left(\hat{\omega}_{(1)} \cos \theta+\hat{\omega}_{(0)}\right) d \phi\right]^{2} \\
& +\left(\frac{k_{i}^{2} / h_{i}+l_{i}}{r}+c_{1}\right) \frac{r}{h_{i}}\left[\left\{d \psi+\left(h_{i} \cos \theta+\chi_{(0)}\right) d \phi\right\}^{2}+h_{i}^{2}\left(\frac{d r^{2}}{r^{2}}+d \theta^{2}+\sin ^{2} \theta d \phi^{2}\right)\right]
\end{aligned}
$$

First, to remove the divergences in the functions $f^{-1}$ and $\omega_{\psi}$, the following conditions must be imposed on the parameters $\left(k_{i}, l_{i}, m_{i}\right)$,

$$
\begin{aligned}
& l_{i}=-\frac{k_{i}^{2}}{h_{i}}, \\
& m_{i}=\frac{k_{i}^{3}}{2 h_{i}^{2}},
\end{aligned}
$$

which gives

$$
\frac{k_{i}^{3}}{h_{i}^{2}}+\frac{3 k_{i} l_{i}}{2 h_{i}}+m_{i}=0, \quad c_{2}=-h_{i} \hat{\omega}_{(1)} .
$$

Introducing the new coordinates $\left(\rho, \psi^{\prime}, \phi^{\prime}\right)$ by

$\rho=2 \sqrt{h_{i} c_{1} r}, \quad \psi^{\prime}=\psi+\chi_{(0)} \phi, \quad \phi^{\prime}=\phi$,

we can obtain the metric near $\boldsymbol{r}=\boldsymbol{r}_{i}$, which is given by 


$$
\begin{aligned}
d s^{2} \simeq & -c_{1}^{-2} d\left[t+c_{2} \psi^{\prime}+\hat{\omega}_{(0)} \phi^{\prime}\right]^{2}+d \rho^{2} \\
& +\frac{\rho^{2}}{4}\left[\left(d \psi^{\prime}+h_{i} \cos \theta d \phi^{\prime}\right)^{2}+d \theta^{2}+\sin ^{2} \theta d \phi^{\prime 2}\right],
\end{aligned}
$$

where to ensure that the metric has the Lorentzian signature we have imposed

$$
h_{i} c_{1}>0, \quad i=2, \ldots, n .
$$

Next, as explained in detail in Ref. [25], to remove the causal violation around each $\boldsymbol{r}_{i}$, we must impose that at $\boldsymbol{r}=\boldsymbol{r}_{i}(i=2, \ldots, n)$

$$
\begin{gathered}
c_{2}=0, \\
\omega_{(0)}=0 .
\end{gathered}
$$

As shown below, the so-called bubble equations (64) automatically guarantee $\hat{\omega}_{(0)}=0$ for all $i=2, \ldots, n$, Therefore, each point $\boldsymbol{r}=\boldsymbol{r}_{i}(i=2, \ldots, n)$ corresponds merely to the coordinate singularities like the origin of the Minkowski spacetime. Thus, we have shown that the points $\boldsymbol{r}=\boldsymbol{r}_{i}$ $(i=2, \ldots, n)$ describe the timelike and regular points.

Finally, we prove $\hat{\omega}_{(0)}=0$ holds at each $\boldsymbol{r}=\boldsymbol{r}_{i}$ for $i=2, \ldots, n$. It can be shown from (58) and (59) that the bubble equations (64) can be written as

$$
\begin{aligned}
0 & =m_{0}-\frac{3}{2} k_{i} l_{0}+h_{0} m_{i}+\sum_{j(\neq i)} \frac{1}{\left|z_{j i}\right|}\left[3 k_{i}^{2} k_{j}+2 k_{i}^{3} h_{j}-\frac{3}{2}\left(k_{i} l_{j}+l_{i} k_{j}+k_{i} l_{i} h_{j}\right)+m_{j}\right] \\
& =m_{0}-\frac{3}{2} k_{i}+h_{0} m_{i}-\frac{n k_{i}^{3}+3 k_{1} k_{i}^{2}-3 l_{1} k_{i}+2 m_{1}}{2 z_{1 i}}+\sum_{2 \leq j(\neq i)} \frac{\left(k_{j}-k_{i}\right)^{3}}{2\left|z_{j i}\right|} .
\end{aligned}
$$

Furthermore, the summation of (66) for $i=2, \ldots, n$ gives

$$
\begin{aligned}
0 & =\sum_{2 \leq j}\left[m_{0}-\frac{3}{2} k_{j}+h_{0} m_{j}-\frac{n k_{j}^{3}+3 k_{1} k_{j}^{2}-3 l_{1} k_{j}+2 m_{1}}{2 z_{1 j}}+\sum_{2 \leq k \neq i)} \frac{\left(k_{k}-k_{j}\right)^{3}}{2\left|z_{k j}\right|}\right] \\
& =\sum_{2 \leq j}\left(m_{0}-\frac{3}{2} k_{j}+h_{0} m_{j}\right)-\sum_{2 \leq j} \frac{n k_{j}^{3}+3 k_{1} k_{j}^{2}-3 l_{1} k_{j}+2 m_{1}}{2 z_{1 j}}
\end{aligned}
$$

where the last term in the first line vanishes by the antisymmetry for $k$ and $j$. From Eqs. (58) and (59), $\hat{\omega}_{(0)}$ is written as

$$
\begin{aligned}
\hat{\omega}_{(0)}= & \sum_{k, j(k, j \neq i, k \neq j)}\left(h_{k} m_{j}+\frac{3}{2} k_{k} l_{j}\right) \frac{z_{j i} z_{k i}}{\left|z_{j i} z_{k i}\right| z_{j k}}+\sum_{j(\neq i)}\left(m_{0} h_{j}+\frac{3}{2} k_{j} l_{0}-h_{0} m_{j}\right) \frac{z_{j i}}{\left|z_{j i}\right|} \\
= & -\sum_{2 \leq j(\neq i)} \frac{n k_{j}^{3}-2 h_{j}^{3} m_{1}-3 h_{j} k_{1} k_{j}^{2}-3 h_{j}^{2} k_{j} l_{1}}{2 h_{j}^{2} z_{j 1}} \frac{z_{j i}}{\left|z_{j i}\right|}+\sum_{2 \leq k, j(k, j \neq i, k \neq j)} \frac{h_{k} k_{j}^{3}-3 h_{j} k_{k} k_{j}^{2}}{2 h_{j}^{2} z_{j k}} \frac{z_{j i} z_{k i}}{\left|z_{j i} z_{k i}\right|}-\left(n m_{0}+\frac{3}{2} l_{0} k_{1}-h_{0} m_{1}\right) \\
& +\sum_{2 \leq j(\neq i)}\left(-m_{0}+\frac{3}{2} k_{j}-h_{0} m_{j}\right) \frac{z_{j i}}{\left|z_{j i}\right|}-\sum_{2 \leq j} \frac{n k_{j}^{3}-2 h_{j}^{3} m_{1}-3 h_{j} k_{1} k_{j}^{2}-3 h_{j}^{2} l_{1} k_{j}}{2 h_{j}^{2} z_{j 1}}-\sum_{2 \leq k, j(k \neq j)} \frac{h_{k} k_{j}^{3}-3 h_{j} k_{k} k_{j}^{2}}{2 h_{j}^{2} z_{k j}} .
\end{aligned}
$$

The third, fifth, and sixth terms of the right-hand side of (68) are combined into

$$
\begin{aligned}
& -\left(n m_{0}+\frac{3}{2} k_{1} l_{0}-h_{0} m_{1}\right)-\sum_{2 \leq j} \frac{n k_{j}^{3}-2 h_{j}^{3} m_{1}-3 h_{j} k_{1} k_{j}^{2}-3 h_{j}^{2} l_{1} k_{j}}{2 h_{j}^{2} z_{j 1}}-\sum_{2 \leq k, j(k \neq j)} \frac{h_{k} k_{j}^{3}-3 h_{j} k_{k} k_{j}^{2}}{2 h_{j}^{2} z_{k j}} \\
& =-\left(n m_{0}+\frac{3}{2} k_{1} l_{0}-h_{0} m_{1}\right)+\sum_{2 \leq j}\left(m_{0}-\frac{3}{2} k_{j} l_{0}+h_{0} m_{j}\right)+\sum_{2 \leq k, j(k \neq j)} \frac{\left(k_{k}-k_{j}\right)^{3}}{4 z_{k j}} \\
& =\sum_{2 \leq j, k(k \neq j)} \frac{\left(k_{k}-k_{j}\right)^{3}}{4 z_{k j}}
\end{aligned}
$$


where we have used Eq. (67) for the second term in the first line and Eq. (28) for the last equality. Next, the summation of the first, second, and fourth terms on the right-hand side of (68) reduces to

$$
\begin{aligned}
& -\sum_{2 \leq j(\neq i)} \frac{n k_{j}^{3}-2 h_{j}^{3} m_{1}-3 h_{j} k_{1} k_{j}^{2}-3 h_{j}^{2} k_{j} l_{1}}{2 h_{j}^{2} z_{j 1}} \frac{z_{j i}}{\left|z_{j i}\right|}+\sum_{2 \leq k, j(k, j \neq i, k \neq j)} \frac{h_{k} k_{j}^{3}-3 h_{j} k_{k} k_{j}^{2}}{2 h_{j}^{2} z_{j k}} \frac{z_{j i} z_{k i}}{\left|z_{j i} z_{k i}\right|}+\sum_{2 \leq j(\neq i)}\left(-m_{0}+\frac{3}{2} k_{j}-h_{0} m_{j}\right) \frac{z_{j i}}{\left|z_{j i}\right|} \\
& =\sum_{2 \leq j \neq i} \frac{z_{j i}}{\left|z_{j i}\right|}\left[\left(-m_{0}+\frac{3}{2} k_{j}-h_{0} m_{j}+\frac{n k_{j}^{3}+3 k_{1} k_{j}^{2}-3 k_{j} l_{1}+2 m_{1}}{2 z_{1 j}}\right)+\sum_{2 \leq k(\neq i, j)} \frac{\left(k_{k}-k_{j}\right)^{3}}{4 z_{j k}} \frac{z_{k i}}{\left|z_{k i}\right|}\right] \\
& =\sum_{2 \leq j \neq i} \frac{z_{j i}}{\left|z_{j i}\right|}\left[\left(\sum_{2 \leq k(\neq j)} \frac{\left(k_{k}-k_{j}\right)^{3}}{2\left|z_{k j}\right|}\right)+\sum_{2 \leq k(\neq i, j)} \frac{\left(k_{k}-k_{j}\right)^{3}}{4 z_{j k}} \frac{z_{k i}}{\left|z_{k i}\right|}\right] \\
& =\sum_{2 \leq j(\neq i)} \frac{z_{j i}}{\left|z_{j i}\right|}\left[\sum_{2 \leq k(\neq j)} \frac{\left(k_{k}-k_{j}\right)^{3}}{2\left|z_{k j}\right|}+\sum_{2 \leq k(\neq i, j)} \frac{\left(k_{k}-k_{j}\right)^{3}}{4 z_{j k}} \frac{z_{k i}}{\left|z_{k i}\right|}\right] \\
& =-\sum_{2 \leq j, k(k \neq j)} \frac{\left(k_{k}-k_{j}\right)^{3}}{4 z_{k j}}
\end{aligned}
$$

where we have used Eq. (67) for the second equality. Thus, the straightforward computations enable us to show that (71) coincides with (69) up to the minus sign. This completes the proof of $\hat{\omega}_{(0)}=0$.

\section{Axis}

The $z$ axis of $\mathbb{E}^{3}$ (i.e., $\left.x=y=0\right)$ in the Gibbons-Hawking space is split into the $(n+1)$ intervals as $I_{-}=$ $\left\{(x, y, z) \mid x=y=0, z<z_{1}\right\}, I_{i}=\left\{(x, y, z) \mid x=y=0, z_{i}<z<z_{i+1}\right\}(i=1, \ldots, n-1)$, and $I_{+}=\{(x, y, z) \mid x=y=0$, $\left.z>z_{n}\right\}$. We find that, on $I_{ \pm}, \hat{\omega}$ vanishes since

$$
\begin{aligned}
\hat{\omega} & =\sum_{k, j(k \neq j)}\left(h_{k} m_{j}+\frac{3}{2} k_{k} l_{j}\right) \hat{\omega}_{k j}-\sum_{j}\left(m_{0} h_{j}+\frac{3}{2} l_{0} k_{j}-h_{0} m_{j}\right) \hat{\omega}_{j}+c d \phi \\
& =\sum_{k, j(k \neq j)}\left(h_{k} m_{j}+\frac{3}{2} k_{k} l_{j}\right) \frac{d \phi}{z_{j k}} \mp \sum_{j}\left(m_{0} h_{j}+\frac{3}{2} l_{0} k_{j}-h_{0} m_{j}\right) d \phi-\sum_{k, j(k \neq j)}\left(h_{k} m_{j}+\frac{3}{2} k_{k} l_{j}\right) \frac{d \phi}{z_{j k}} \\
& =\mp \sum_{j}\left(m_{0} h_{j}+\frac{3}{2} l_{0} k_{j}-h_{0} m_{j}\right) d \phi \\
& =0,
\end{aligned}
$$

where we have used Eqs. (29) and (28), respectively, in the second equality and the last equality.

For $z \in I_{i}(i=1,2, \ldots, n-1)$, we find

$$
\begin{aligned}
\left.\hat{\omega}_{\phi}\right|_{I_{i}} & =\hat{\omega}_{\phi}\left(\rho=0, z_{i}<z<z_{i+1}\right) \\
& =\left.\hat{\omega}_{\phi}\right|_{r=r_{i}}(\theta=0) \\
& =\hat{\omega}_{(1)}+\hat{\omega}_{(0)} \\
& =-c_{2} \\
& =0
\end{aligned}
$$

where we have used the fact that $\hat{\omega}$ is constant on $I_{i}$ in the second equality and Eq. (53) in the third equality. Furthermore, we have used Eqs. (60) and (64), respectively, in the fourth equality and last equality. It hence turns out that $\hat{\omega}=0$ holds at each interval, which proves that no Dirac-Misner string pathologies exist throughout the spacetime.

In turns, we prove the absence of orbifold singularities. On $I_{ \pm}$, we have

$$
\chi= \pm d \phi,
$$

whereas on $I_{i}$, we have 


$$
\begin{aligned}
\chi & =\left(n \frac{z-z_{1}}{\left|z-z_{1}\right|}-\sum_{2 \leq j \leq i} \frac{z-z_{j}}{\left|z-z_{j}\right|}-\sum_{i+1 \leq j \leq n-1} \frac{z-z_{j}}{\left|z-z_{j}\right|}\right) d \phi \\
& =(2 n-2 i+1) d \phi .
\end{aligned}
$$

Therefore, the two-dimensional $(\phi, \psi)$ part of the metric on the intervals $I_{ \pm}$and $I_{i}$ takes the following simple form:

$$
d s_{2}^{2}=\left(-f^{2} \omega_{\psi}+f^{-1} H^{-1}\right)\left(d \psi+\chi_{\phi} d \phi\right)^{2} .
$$

In the analysis of orbifold singularities, it is more useful to consider in the coordinate basis $\left(\partial_{\phi_{1}}, \partial_{\phi_{2}}\right)$ of the periodicity of $2 \pi$, in $\left(\partial_{\phi}, \partial_{\psi}\right)$, where these coordinates are defined by $\phi_{1}:=(\psi+\phi) / 2$ and $\phi_{2}:=(\psi-\phi) / 2$. From Eq. (80), we see that the Killing vector $v:=\partial_{\phi}-\chi_{\phi} \partial_{\psi}$ vanishes on each interval. Therefore, we can obtain the rod structure is given by the following:

(i) On the interval $I_{+}$, the Killing vector $v_{+}:=\partial_{\phi}-$ $\partial_{\psi}=(0,-1)$ vanishes.

(ii) On each interval $I_{i}(i=1, \ldots, n-1)$, the Killing vector $v_{i}:=\partial_{\phi}-(2 n-2 i+1) \partial_{\psi}=(i-n, i-n-1)$ vanishes.

(iii) On the interval $I_{-}$, the Killing vector $v_{-}:=\partial_{\phi}+$ $\partial_{\psi}=(1,0)$ vanishes.

From these, we can observe that the Killing vectors $v_{ \pm}$and $v_{i}$ on the intervals satisfy

$$
\operatorname{det}\left(v_{+}^{T}, v_{n-1}^{T}\right)=-1, \operatorname{det}\left(v_{i}^{T}, v_{i-1}^{T}\right)=-1,
$$

with

$$
\operatorname{det}\left(v_{1}^{T}, v_{-}^{T}\right)=n
$$

Equation (81) means that there exist no orbifold singularities at adjacent intervals $z=z_{i}(1 \leq i \leq n)$ [13], and Eq. (82) shows that the spatial topology of the horizon is the lens space $L(n, 1)=S^{3} / \mathbb{Z}_{n}$.

\section{PHYSICAL PROPERTIES}

Since appropriate boundary conditions are given in the last section, we can now investigate several physical properties of the solution obtained in Sec. II. To do so, we can consider the physical conserved charges from two points of view, in the five-dimensional minimal supergravity and in the dimensionally reduced four-dimensional theory, which leads to a massless axion and a dilaton coupled to gravity and two $U(1)$ gauge fields, one of which has Chern-Simon coupling. Here, let us take the five-dimensional point of view for the simplicity. Since at infinity $r \rightarrow \infty$ the spacetime asymptotically behaves as an $S^{1}$ fiber bundle over fourdimensional Minkowski spacetime, the metric of which can be written as $g_{\mu \nu} \simeq \eta_{\mu \nu}+h_{\mu \nu}$ in Cartesian coordinates, the Arnowitt-Deser-Misner (ADM) mass and ADM (angular) momentum can be computed. Following the notations in Ref. [42], we can express the $2 \times 2$ ADM stress tensor as

$$
\begin{aligned}
T_{a b}= & \frac{1}{16 \pi G_{5}} \int_{S_{\infty}} d \Omega_{S^{2}}^{2} r^{2} n^{i}\left[\eta_{a b}\left(\partial_{i} h_{c}^{c}-\partial_{j} h_{i}^{j}\right)-\partial_{i} h_{a b}\right], \\
& (a, b, c=\bar{t}, \bar{w}, i, j=x, y, z)
\end{aligned}
$$

where $d \Omega_{S^{2}}^{2}$ is a volume element of a two-dimensional sphere with unit radius and $n^{i}$ is the radial unit normal vector. In terms of the stress tensor, we have the ADM mass and $\mathrm{ADM}$ (angular) momentum along the fifth dimension $\partial / \partial \bar{w}$, respectively, as

$$
\begin{aligned}
M & =\int d \bar{w} T_{\bar{t} \bar{t}}=\frac{\pi R_{k}}{2 G_{5}} \frac{\left(1-2 h_{0} m_{0}^{2}\right) \sum_{i} h_{i}+h_{0}\left(3 \sum_{i} l_{i}-3 m_{0} \sum_{i} k_{i}-2 h_{0} m_{0} \sum_{i} m_{i}\right)}{1-h_{0} m_{0}^{2}} \\
P & =\int d \bar{w} T_{\bar{t} \bar{w}}=\frac{\pi R_{k}}{4 G_{5}} \frac{2 m_{0} \sum_{i} h_{i}-6 h_{0} m_{0} \sum_{i} l_{i}+\left(1+h_{0} m_{0}^{2}\right)\left(3 \sum_{i} k_{i}+2 h_{0} \sum m_{i}\right)}{1-h_{0} m_{0}^{2}} .
\end{aligned}
$$

Moreover, the angular momentum along $\partial / \partial \phi$ can be obtained as

$$
J_{\phi}=\frac{\pi R_{5}}{G_{5}} \sum_{i}\left(m_{0} h_{i}+\frac{3}{2} k_{i}-h_{0} m_{0}\right) z_{i} .
$$

As pointed out in Refs. [24,25], let us note that the asymptotically flat supersymmetric black lens must have two nonzero-angular momenta. Now, we would like to see whether the Kaluza-Klein black lens obtained here allows two zero-angular momenta or not, in particular, for the simplest case of $n=2$. From Eq. (64), $z_{21}$ can be written in terms of the other parameters, as

$$
z_{21}=\frac{2 k_{2}^{3}+3 k_{1} k_{2}^{2}-3 l_{1} k_{2}+2 m_{1}}{-2 h_{0} k_{2}^{3}+6 k_{2}+3 k_{1}-2 h_{0} m_{1}},
$$

and the substitution of this into the inequality (63) yields

$$
\begin{aligned}
& \left(c_{1}=\right) 1-h_{0} k_{2}^{2} \\
& +\frac{\left(-2 h_{0} k_{2}^{3}+6 k_{2}+3 k_{1}-2 h_{0} m_{1}\right)\left(l_{1}-2 k_{2}^{2}-2 k_{1} k_{2}\right)}{2 k_{2}^{3}+3 k_{1} k_{2}^{2}-3 l_{1} k_{2}+2 m_{1}}<0 .
\end{aligned}
$$

The solid curve in Fig. 1 shows the plots of $P=0$ in the $\left(k_{1}, k_{2}\right)$ plane for $n=2, z_{1}=0$, and $h_{0}=m_{1}=l_{1}=1$. 


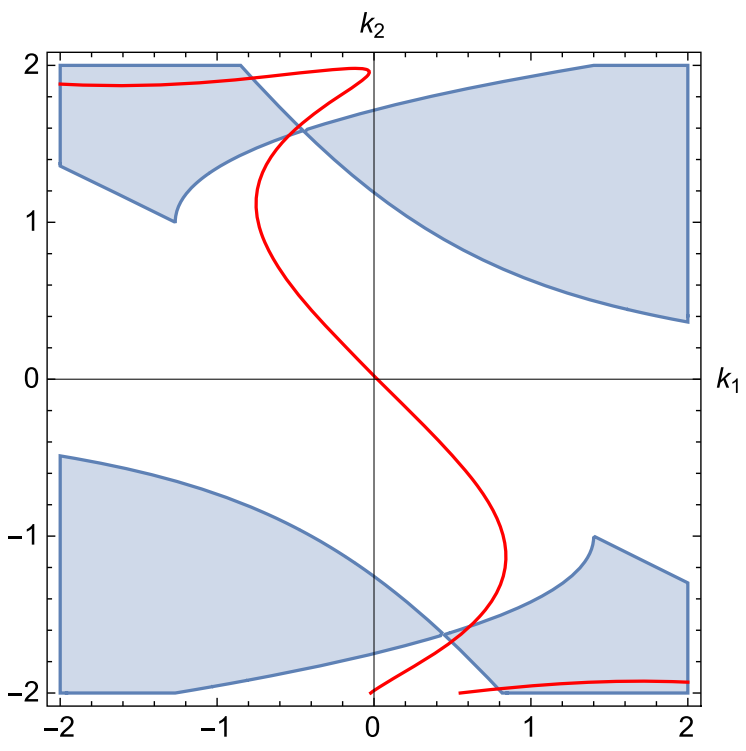

FIG. 1. Plots of $P=0$ in a $\left(k_{1}, k_{2}\right)$ plane for $n=2, z_{1}=0$, $h_{0}=m_{1}=l_{1}=1$. The shaded regions and the curve denote the regions of $R_{1}^{2}>0, R_{2}^{2}>0, c_{1}<0, z_{21}>0$, and $P=0$, respectively. The (angular) momentum $P$ vanishes where the curve crosses on the shaded region.

The shaded portions in this figure present the region such that all of the inequalities $R_{1}^{2}>0, R_{2}^{2}>0, z_{21}>0$, and $c_{1}<0$ are satisfied. It can be seen from this figure that there indeed exists a parameter region in which $P=0$ can be realized. Moreover, it can be shown from Eq. (28) that for $n=2$ the angular momentum $J_{\phi}$ vanishes in the choice of the parameter $z_{1}=0$. Therefore, at least, for $n=2$, in contrast to the result of the asymptotically flat supersymmetric black lens, we can see that there exists a case in which both of angular momenta $\left(J_{\phi}, P\right)$ vanish.

The interval $I_{1}$ is topologically a disk, and the $(n-2)$ intervals $I_{i}(i=2, \ldots, n-1)$ are a two-dimensional sphere. The magnetic fluxes through $I_{i}$ that are defined by

$$
q\left[I_{i}\right]:=\frac{1}{4 \pi} \int_{I_{i}} F
$$

are computed as

$$
\begin{aligned}
& q\left[I_{1}\right]=\frac{\sqrt{3}}{2}\left[\frac{k_{1} l_{1}}{2\left(k_{1}^{2}+n l_{1}\right)}-k_{2}\right], \\
& q\left[I_{i}\right]=\frac{\sqrt{3}}{2}\left(k_{i}-k_{i+1}\right) \quad(i=2, \ldots n-1) .
\end{aligned}
$$

In particular, the first term in $q\left[I_{1}\right]$ gives the contribution from the horizon, whereas the second term and each term in $q\left[I_{i}\right](i=2, \ldots, n)$ come from $\boldsymbol{r}=\boldsymbol{r}_{i}(i=2, \ldots, n)$. The expression (88) for the magnetic fluxes are exactly the same as for the asymptotically flat black lens with the horizon topology of $L(n, 1)$ in Ref. [25]. As shown in Refs. [24,25], for the asymptotically flat supersymmetric black lenses, the existence of the magnetic fluxes plays an essential role in supporting the horizon of the lens space topology. On the contrary, it can be shown that this is not true for the Kaluza-Klein supersymmetric black lens obtained here. In turns, we consider whether the Kaluza-Klein black lens also prohibits $\left(q\left[I_{1}\right], \ldots, q\left[I_{n-1}\right]\right)=(0, \ldots, 0)$. For $k_{2}=k_{1} l_{1} /\left[2\left(k_{1}^{2}+n l_{1}\right)\right], \quad k_{i}=k_{i+1}(i=2, \ldots, n-1)$, all magnetic fluxes $q\left[I_{i}\right](i=1, \ldots, n-1)$ vanish. In the choice of these parameters, the condition (63) can be simply written as

$$
-\frac{n l_{1}^{2}\left(3 k_{1}^{2}+4 n l_{1}\right)}{4\left(k_{1}^{2}+n l_{1}\right)^{2}}>\left(1-h_{0} k_{i}^{2}\right) z_{i 1}, \quad i=2, \ldots, n .
$$

As exactly proved in Ref. [25] for the asymptotically flat black lens, which can be realized by putting $h_{0}=m_{1}=0$, these inequalities cannot be satisfied, since the left-hand side is nonpositive by Eq. (48) but the right-hand side must be positive from our assumption. However, for the KaluzaKlein black lens with $h_{0}>0$, the right-hand side can be negative due to the existence of the constant $h_{0}$, which corresponds to the size of an extra dimension at infinity. In fact, we can see from Fig. 2 that the magnetic flux can vanish, at least, for $n=2$. The solid curve in Fig. 2 denotes the plots of $q\left[I_{1}\right]=0$ in the $\left(k_{1}, k_{2}\right)$ plane for $n=2$, $z_{1}=0, h_{0}=10^{5}, m_{1}=10^{-4}$, and $l_{1}=1$, and the two separated shaded portions are the regions such that all of the inequalities $R_{1}^{2}>0, R_{2}^{2}>0, z_{21}>0$, and $c_{1}<0$ are satisfied. Therefore, the magnetic flux $q\left[I_{1}\right]$ vanishes on the solid curve in the shaded regions. Thus, in contrast to the

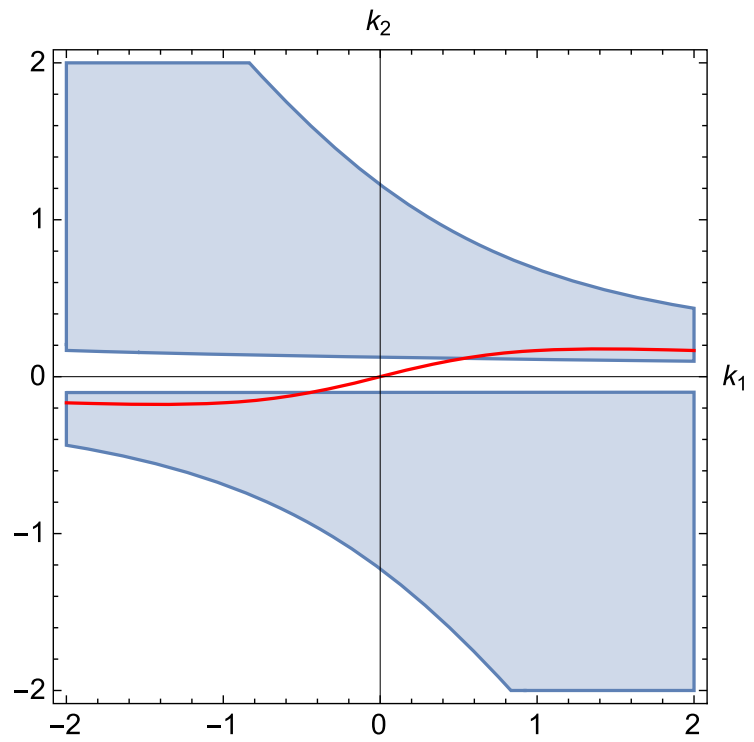

FIG. 2. Plots of $q\left[I_{1}\right]=0$ in the $\left(k_{1}, k_{2}\right)$ plane for $n=2$, $z_{1}=0, h_{0}=10^{5}, m_{1}=10^{-4}$, and $l_{1}=1$. The shaded regions and the curve denote the regions of $R_{1}^{2}>0, R_{2}^{2}>0, z_{21}>0$ $c_{1}<0$, and $q\left[I_{1}\right]=0$, respectively. The magnetic flux $q\left[I_{1}\right]$ vanishes on the curve in the shaded regions. 
asymptotically flat supersymmetric black lens, the magnetic flux can vanish for the Kaluza-Klein supersymmetric black lens.

\section{SUMMARY}

In this work, we have constructed a biaxisymmetric Kaluza-Klein black lens solution as a supersymmetric solution in the bosonic sector of the five-dimensional minimal supergravity. We have shown that the spacetime has a degenerate Killing horizon with the spatial cross section of the lens topology of $L(n, 1)=S^{3} / \mathbb{Z}_{n}$ and also computed the mass, two angular momenta, and $(n-1)$ magnetic fluxes. When the compactification radius of the extra dimension becomes infinite, this solution exactly coincides with the asymptotically flat black lens in the previous work $[24,25]$.
For the asymptotically flat supersymmetric black lens in Refs. [24,25], which can be obtained by taking the limit $h_{0} \rightarrow 0$, a pair of angular momenta cannot vanish, whereas for the Kaluza-Klein black lens in this paper, both of them can vanish at least for $n=2$. For the asymptotically flat black lens, the existence of the magnetic fluxes plays an essential role in supporting the horizon of the black lens, whereas for the Kaluza-Klein black lens obtained in this paper, this cannot be applied since the magnetic flux vanishes, at least, for $n=2$.

\section{ACKNOWLEDGMENTS}

This work was supported by the Grant-in-Aid for Scientific Research (C) (Grant No. 17K05452) from the Japan Society for the Promotion of Science.
[1] J. P. Gauntlett, J. B. Gutowski, C. M. Hull, S. Pakis, and H. S. Reall, All supersymmetric solutions of minimal supergravity in five- dimensions, Classical Quantum Gravity 20, 4587 (2003).

[2] S. Mizoguchi and S. Tomizawa, New approach to solution generation using $\mathrm{SL}(2, \mathrm{R})$-duality of a dimensionally reduced space in five-dimensional minimal supergravity and new black holes, Phys. Rev. D 84, 104009 (2011).

[3] S. Mizoguchi and S. Tomizawa, Flipped $S L(2, R)$ duality in five-dimensional supergravity, Phys. Rev. D 86, 024022 (2012).

[4] S. Tomizawa and S. Mizoguchi, General Kaluza-Klein black holes with all six independent charges in fivedimensional minimal supergravity, Phys. Rev. D 87, 024027 (2013).

[5] J. Ford, S. Giusto, A. Peet, and A. Saxena, Reduction without reduction: Adding KK-monopoles to five dimensional stationary axisymmetric solutions, Classical Quantum Gravity 25, 075014 (2008).

[6] S. Giusto, S. F. Ross, and A. Saxena, Non-supersymmetric microstates of the D1-D5-KK system, J. High Energy Phys. 12 (2007) 065.

[7] G. Compere, S. de Buyl, E. Jamsin, and A. Virmani, G2 dualities in $D=5$ supergravity and black strings, Classical Quantum Gravity 26, 125016 (2009).

[8] A. Bouchareb, G. Clement, C. M. Chen, D. V. Gal'tsov, N. G. Scherbluk, and T. Wolf, G(2) generating technique for minimal $D=5$ supergravity and black rings, Phys. Rev. D 76, 104032 (2007); Erratum 78, 029901 (2008).

[9] D. V. Gal'tsov and N. G. Scherbluk, Improved generating technique for $D=5$ supergravities and squashed KaluzaKlein black holes, Phys. Rev. D 79, 064020 (2009).

[10] S. Tomizawa, Y. Yasui, and A. Ishibashi, Uniqueness theorem for charged rotating black holes in fivedimensional minimal supergravity, Phys. Rev. D 79, 124023 (2009).
[11] S. Tomizawa, Y. Yasui, and A. Ishibashi, Uniqueness theorem for charged dipole rings in five-dimensional minimal supergravity, Phys. Rev. D 81, 084037 (2010).

[12] S. Tomizawa, Uniqueness theorems for Kaluza-Klein black holes in five-dimensional minimal supergravity, Phys. Rev. D 82, 104047 (2010).

[13] S. Hollands and S. Yazadjiev, Uniqueness theorem for 5dimensional black holes with two axial Killing fields, Commun. Math. Phys. 283, 749 (2008).

[14] S. Hollands, J. Holland, and A. Ishibashi, Further restrictions on the topology of stationary black holes in five dimensions, Ann. Inst. Henri Poincaré 12, 279 (2011).

[15] M. 1. Cai and G. J. Galloway, On the Topology and area of higher dimensional black holes, Classical Quantum Gravity 18, 2707 (2001).

[16] G. J. Galloway and R. Schoen, A generalization of Hawking's black hole topology theorem to higher dimensions, Commun. Math. Phys. 266, 571 (2006).

[17] V. Breunhölder and J. Lucietti, Moduli space of supersymmetric solitons and black holes in five dimensions, arXiv:1712.07092.

[18] F. R. Tangherlini, Schwarzschild field in n dimensions and the dimensionality of space problem, Nuovo Cimento 27, 636 (1963).

[19] R. C. Myers and M. J. Perry, Black holes in higher dimensional space-times, Ann. Phys. (N.Y.) 172, 304 (1986).

[20] R. Emparan and H.S. Reall, A Rotating Black Ring Solution in Five-Dimensions, Phys. Rev. Lett. 88, 101101 (2002).

[21] A. A. Pomeransky and R. A. Sen'kov, Black ring with two angular momenta, arXiv:hep-th/0612005.

[22] J. Evslin, Geometric engineering 5d black holes with rod diagrams, J. High Energy Phys. 09 (2008) 004.

[23] Y. Chen and E. Teo, A rotating black lens solution in five dimensions, Phys. Rev. D 78, 064062 (2008). 
[24] H. K. Kunduri and J. Lucietti, Supersymmetric Black Holes with Lens-Space Topology, Phys. Rev. Lett. 113, 211101 (2014).

[25] S. Tomizawa and M. Nozawa, Supersymmetric black lenses in five dimensions, Phys. Rev. D 94, 044037 (2016).

[26] S. Tomizawa and T. Okuda, Asymptotically flat multiblack lenses, Phys. Rev. D 95, 064021 (2017).

[27] S. Tomizawa, Charged black lens in de Sitter space, Phys. Rev. D 97, 044001 (2018).

[28] S. Tomizawa and H. Ishihara, Exact solutions of higher dimensional black holes, Prog. Theor. Phys. Suppl. 189, 7 (2011).

[29] P. Dobiasch and D. Maison, Stationary, spherically symmetric solutions of Jordan's unified theory of gravity and electromagnetism, Gen. Relativ. Gravit. 14, 231 (1982).

[30] D. Rasheed, The rotating dyonic black holes of KaluzaKlein theory, Nucl. Phys. B454, 379 (1995).

[31] H. Ishihara and K. Matsuno, Kaluza-Klein black holes with squashed horizons, Prog. Theor. Phys. 116, 417 (2006).

[32] H. Ishihara, M. Kimura, K. Matsuno, and S. Tomizawa, Kaluza-Klein multi-black holes in five-dimensional Einstein-Maxwell theory, Classical Quantum Gravity 23, 6919 (2006).

[33] T. Nakagawa, H. Ishihara, K. Matsuno, and S. Tomizawa, Charged rotating Kaluza-Klein black holes in five dimensions, Phys. Rev. D 77, 044040 (2008).
[34] K. Matsuno, H. Ishihara, T. Nakagawa, and S. Tomizawa, Rotating Kaluza-Klein multi-black holes with Godel parameter, Phys. Rev. D 78, 064016 (2008).

[35] S. Tomizawa, H. Ishihara, K. Matsuno, and T. Nakagawa, Squashed Kerr-Godel black holes: Kaluza-Klein black holes with rotations of black hole and universe, Prog. Theor. Phys. 121, 823 (2009).

[36] S. Tomizawa and A. Ishibashi, Charged black holes in a rotating Gross-Perry-Sorkin monopole background, Classical Quantum Gravity 25, 245007 (2008).

[37] G. W. Gibbons and S. W. Hawking, Gravitational multiinstantons, Phys. Lett. B 78, 430 (1978).

[38] M. Dunajski and S. A. Hartnoll, Einstein-Maxwell gravitational instantons and five dimensional solitonic strings, Classical Quantum Gravity 24, 1841 (2007).

[39] I. Bena, P. Kraus, and N. P. Warner, Black rings in TaubNUT, Phys. Rev. D 72, 084019 (2005).

[40] J. C. Breckenridge, R. C. Myers, A. W. Peet, and C. Vafa, D-branes and spinning black holes, Phys. Lett. B 391, 93 (1997).

[41] A. H. Chamseddine, S. Ferrara, G. W. Gibbons, and R. Kallosh, Enhancement of supersymmetry near 5-d black hole horizon, Phys. Rev. D 55, 3647 (1997).

[42] H. Elvang, R. Emparan, D. Mateos, and H. S. Reall, Supersymmetric 4-D rotating black holes from 5-D black rings, J. High Energy Phys. 08 (2005) 042. 